\title{
Timed-Release Cryptography
}

\author{
Wenbo Mao \\ Hewlett-Packard Laboratories, \\ Filton Road, Stoke Gifford, Bristol BS34 8QZ, United Kingdom \\ wm@hplb.hpl.hp.com
}

\begin{abstract}
Let $n$ be a large composite number. Without factoring $n$, the computation of $a^{2^{t}}(\bmod n)$ given $a, t$ with $\operatorname{gcd}(a, n)=1$ and $t<n$ can be done in $t$ squarings modulo $n$. For $t \ll n$ (e.g., $n>2^{1024}$ and $t<2^{100}$ ), no lower complexity than $t$ squarings is known to fulfill this task. Rivest et al suggested to use such constructions as good candidates for realising timed-release crypto problems.

We argue the necessity for a zero-knowledge proof of the correctness of such constructions and propose the first practically efficient protocol for a realisation. Our protocol proves, in $\log _{2} t$ standard crypto operations, the correctness of $\left(a^{e}\right)^{2^{t}}(\bmod n)$ with respect to $a^{e}$ where $e$ is an RSA encryption exponent. With such a proof, a Timed-release Encryption of a message $M$ can be given as $a^{2^{t}} M(\bmod n)$ with the assertion that the correct decryption of the RSA ciphertext $M^{e}(\bmod n)$ can be obtained by performing $t$ squarings modulo $n$ starting from $a$. Timed-release RSA signatures can be constructed analogously.
\end{abstract}

Keywords Timed-release cryptography, Time-lock puzzles, Non-parallelisability, Efficient zero-knowledge protocols.

\section{Introduction}

Let $n$ be a large composite natural number. Given $t<n$ and $\operatorname{gcd}(a, n)=1$, without factoring $n$, the validation of

$$
X \equiv a^{2^{t}}(\bmod n)
$$

can be done in $t$ squarings mod $n$. However if $\phi(n)$ (Euler's phi function of $n$ ) is known, then the job can be completed in $O(\log n)$ multiplications via the following two steps:

$$
\begin{gathered}
u \stackrel{\text { def }}{=} 2^{t}(\bmod \phi(n)), \\
X \stackrel{\text { def }}{=} a^{u}(\bmod n) .
\end{gathered}
$$

For $t \ll n$ (e.g., $n>2^{1024}$ and $t<2^{100}$ ), it can be anticipated that factoring of $n$ (and hence computing $\phi(n)$ for performing the above steps) will be much more difficult than performing $t$ squarings. Under this condition we do not know any other method which, without using the factorisation of $n$, can compute

S. Vaudenay and A. Youssef (Eds.): SAC 2001, LNCS 2259, pp. 342 3572001.

(C) Springer-Verlag Berlin Heidelberg 2001 
$a^{2^{t}}(\bmod n)$ in time less than $t$ squarings. Moreover, because each squaring can only be performed on the result of the previous squaring, it is not known how to speedup the $t$ squarings via parallelisation of multiple processors. Parallelisation of each squaring step cannot achieve a great deal of speedup since a squaring step only needs a trivial computational resource and so any non-trivial scale of parallelisation of a squaring step is likely to be penalised by communication delays among the processors.

These properties suggest that the following language (notice that each element in the language associates a non-secret natural number $t$ )

$$
L(a, n)=\left\{a^{2^{t}}(\bmod n) \mid \operatorname{gcd}(a, n)=1, t=1,2, \ldots,\right\}
$$

forms a good candidate for the realisation of timed-release crypto problems. Rivest, Shamir and Wagner pioneered the use of this language in a time-lock puzzle scheme [11. In their scheme a puzzle is a triple $(t, a, n)$ and the instruction for finding its solution is to perform $t$ squarings mod $n$ starting from $a$ which leads to $a^{2^{t}}(\bmod n)$. A puzzle maker, with the factorisation knowledge of $n$, can construct a puzzle efficiently using the steps in (2) and (3) and can fine tune the difficulty for finding the solution by choosing $t$ in a vast range. For instance, the MIT Laboratory for Computer Science (LCS) has implemented the timelock puzzle of Rivest et al into "The LCS35 Time Capsule Crypto-Puzzle" and started its solving routine on 4th April 1999. It is estimated that the solution to the LCS35 Time Capsule Crypto-Puzzle will be found in 35 years from 1999, or on the 70 years from the inception of the MIT-LCS [10]. (Though we will discuss a problem of this puzzle in $\S 1.2$.)

\subsection{Applications}

Boneh and Naor used a sub-language of $L(a, n)$ (details to be discussed in $\S 1.2$ ) and constructed a timed-release crypto primitive which they called "timed commitments" 3. Besides several suggested applications they suggested an interesting use of their primitive for solving a long-standing problem in fair contract signing. A previous solution (due to Damgård [6]) for fair contract signing between two remote and mutually distrusted parties is to let them exchange signatures of a contract via gradual release of a secret. A major drawback with that solution is that it only provides a weak fairness. Let us describe this weakness by using, for example, a discrete-logarithm based signature scheme. A signature being gradually released relates to a series of discrete logarithm problems with the discrete logarithm values having gradually decreasing magnitudes. Sooner or later before the two parties completes their exchange, one of them may find himself in a position of extracting a discrete logarithm which is sufficiently small with respect to his computational resource. It is well-known (e.g., the work of van Oorschot and Wiener on the parallelised rho method [13]) that parallelisation is effective for extracting small discrete logarithms. So the resourceful party (one who is able to afford vast parallelisation) can abort the exchange at that point and wins an advanced position unfairly. Boneh and Naor suggested to seal 
signatures under exchange using elements in $L(a, n)$. Recall the aforementioned non-parallelisable property for re-constructing the elements in $L(a, n)$, a roughly equal time can be imposed for both parties to open the sealed signatures regardless of their difference (maybe vast) in computing resources. In this way, they argued that strong fairness for contract signing can be achieved.

Rivest et al suggested several other applications of timed-release cryptography [11:

- A bidder in an auction wants to seal his bid so that it can only be opened after the bidding period is closed.

- A homeowner wants to give his mortgage holder a series of encrypted mortgage payments. These might be encrypted digital cash with different decryption dates, so that one payment becomes decryptable (and thus usable by the bank) at the beginning of each successive month.

- A key-escrow scheme can be based on timed-release crypto, so that the government can get the message keys, but only after a fixed, pre-determined period.

- An individual wants to encrypt his diaries so that they are only decryptable after fifty years (when the individual may have forgotten the decryption key).

\subsection{Previous Work and Unsolved Problem}

With the nice properties of $L(a, n)$ we are only half way to the realisation of timed-release cryptography. In most imaginable applications where timed-release crypto may play a role, it is necessary for a problem constructor to prove (ideally in zero-knowledge) the correct construction of the problem. For example, without a correctness proof, the strong fairness property of the fair-exchange application is actually absent.

From the problem's membership in NP we know that there exists a zeroknowledge proof for a membership assertion regarding language $L(a, n)$. Such a proof can be constructed via a general method (e.g., the work of Goldreich et al [9]). However, the performance of a zero-knowledge proof in a general construction is not suitable for practical use. By the performance for practical use we mean an efficiency measured by a small polynomial in some typical parameters (e.g., the bit length of $n$ ). To our knowledge, there exists no practically efficient zero-knowledge protocols for proving the general case of the membership in $L(a, n)$.

Boneh and Naor constructed a practically efficient protocol for proving membership in a sub-language of $L(a, n)$ where $t=2^{k}$ with $k$ being any natural number. The time control that the elements in this sub-language can offer has the granularity 2 . We know that the time complexity in bit operation for performing one squaring modulo $n$ can be expressed by the lowest known result of $c \cdot \log n \cdot \log \log n$ (where $c>1$ is a machine dependent value, a faster machine has a smaller $c$ ) if FFT (fast Fourier transform) is used for the implementation of squaring. Thus, the time complexity for computing elements in this sub-language is the step function

$$
2^{k} \cdot c \cdot \log n \cdot \log \log n
$$


which has a fast increasing step when $k$ gets large. Boneh and Naor envisioned $k \in[30, \ldots, 50]$ for typical cases in applications. While it is evident that $k$ decreasing from 30 downwards will quickly trivialise a timed-release crypto problem as $2^{30}$ is already at the level of a small polynomial in the secure bit length of $n$ (usually $2^{10}$ ), a $k$ increasing from 30 upwards will harden the problem in such increasingly giant steps that imaginable services (e.g., the strong fairness for gradual disclosure of secret proposed in 3]) will quickly become unattractive or unusable. Taking the LCS35 Time Capsule for example, let the 35-year-openingtime capsule be in that sub-language (so the correctness can be efficiently proved with the protocol in 3]), then the only other elements in that sub-language with opening times close to 35 years will be 17.5 years and 70 years. We should notice that there is no hope to try to tune the size of $n$ as a means of tuning the time complexity since changing $c \cdot \log n \cdot \log \log n$ will have little impact on the above giant step function.

Boneh and Naor expressed a desire for a finer time-control ratio than 2 and sketched a method to obtain a finer ratio with $t_{0}=1$ and $t_{i}=t_{i-1}+t_{i-2}$ for $i=1, \ldots, k$. This method of reducing the ratio renders the ratio being bounded below by $\alpha=\frac{1+\sqrt{5}}{2}(\approx 1.618)$ while increasing the number of proof rounds from $k$ to $\log _{\alpha} k$. They further mentioned that smaller values can be obtained by other such recurrences. It seems to us that if some recurrence method similar to above is used, then with ratio $\rightarrow 1$ ( 1 is the ideal ratio and will be that for our case), the number of proof rounds $\log _{\text {ratio }} k \rightarrow \infty$. So their suggested methods for reducing the time-control ratio are not practical for obtaining a desirable ratio.

The Time-Lock-Puzzle work of Rivest et al [11] did not provide a method for proving the correct construction of a timed-release crypto problem.

\subsection{Our Work}

We construct the first practically efficient zero-knowledge proof protocol for demonstrating the membership in $L(a, n)$ which runs in $\log _{2} t$ steps, each an exponentiation modulo $n$, or $O\left(\log _{2} t \cdot\left(\log _{2} n\right)^{3}\right)$ bit operations in total (without using FFT). This efficiency suits practical uses. The membership demonstration can be conducted in terms of $\left(a^{e}\right)^{2^{t}}(\bmod n) \in L\left(a^{e}, n\right)$ on given $a, a^{e}$ and $t$, where $e$ is an RSA encryption exponent. Then we are able to provide two timedrelease crypto primitives, one for timed release of a message, and the other for timed release of an RSA signature. In the former, a message $M$ can be sealed in $a^{2^{t}} M(\bmod n)$, and the established membership asserts that the correct decryption of the RSA ciphertext $M^{e}(\bmod n)$ can be obtained by performing $t$ squarings modulo $n$ starting from $a$. The latter primitive can be constructed analogously.

Our schemes provide general methods for the use of timed-release cryptography. 


\subsection{Organisation}

In the next section we agree on the notation to be used in the paper. In Section 3 we construct general methods for timed-release cryptography based on proven membership in $L(a, n)$. In Section 4 we construct our membership proof protocol working with an RSA modulus of a safe-prime structure. In Section 5 we will discuss how to generalise our result to working with a general form of composite modulus.

\section{Notation}

Throughout the paper we use the following notation. $\mathbb{Z}_{n}$ denotes the ring of integers modulo $n$. $\mathbb{Z}_{n}^{*}$ denotes the multiplicative group of integers modulo $n . \phi(n)$ denotes Euler's phi function of $n$, which is the order, i.e., the number of elements, of the group $\mathbb{Z}_{n}^{*}$. For an element $a \in \mathbb{Z}_{n}^{*}, \operatorname{ord}_{n}(a)$ denotes the multiplicative order modulo $n$ of $a$, which is the least index $i$ satisfying $a^{i} \equiv 1(\bmod n) ;\langle a\rangle$ denotes the subgroup generated by $a ;\left(\frac{x}{n}\right)$ denotes the Jacobi symbol of $x \bmod n$. We denote by $J_{+}(n)$ the subset of $\mathbb{Z}_{n}^{*}$ containing the elements of the positive Jacobi symbol. For integers $a, b$, we denote by $\operatorname{gcd}(a, b)$ the greatest common divisor of $a$ and $b$. For a real number $r$, we denote by $\lfloor r\rfloor$ the floor of $r$, i.e., $r$ rounded down to the nearest integer.

\section{Timed-Release Crypto with Proven Membership in $L(a, n)$}

Let Alice be the constructor of a timed-release crypto problem. She begins with constructing a composite natural number $n=p q$ where $p$ and $q$ are two distinct odd prime numbers. Define

$$
\begin{gathered}
a(t) \stackrel{\text { def }}{=} a^{2^{t}}(\bmod n), \\
a^{e}(t) \stackrel{\text { def }}{=}(a(t))^{e}(\bmod n),
\end{gathered}
$$

where $e$ is a fixed natural number relatively prime to $\phi(n)$ (in the position of an RSA public exponent), and $a \not \equiv \pm 1(\bmod n)$ is a random element in $\mathbb{Z}_{n}^{*}$. Alice can construct $a(t)$ using the steps in (2) and (3).

The following security requirements should be in place: $n$ should be so constructed that $\operatorname{ord}_{\phi(n)}(2)$ is sufficiently large, and $a$ should be so chosen that $\operatorname{ord}_{n}(a)$ is sufficiently large. Here, "sufficiently large" means "much larger than $t$ " for the largest possible $t$ that the system should accommodate.

In the remainder of this section, we assume that Alice has proven to Bob, the verifier, the following membership status (using the protocol in $\S 4$ ):

$$
a^{e}(t) \in L\left(a^{e}, n\right)
$$


Clearly, with $e$ co-prime to $\phi(n)$, this is equivalent to another membership status:

$$
a(t) \in L(a, n) .
$$

However in the latter case $a(t)$ is (temporarily) unavailable to Bob due to the difficulty of extracting the $e$-th root (of $a^{e}(t)$ ) in the RSA group.

\subsection{Timed-Release of an Encrypted Message}

For message $M<n$, to make it decryptable in time $t$, Alice can construct a "timed encryption":

$$
T E(M, t) \stackrel{\text { def }}{=} a(t) M(\bmod n) .
$$

Let Bob be given the tuple $\left(T E(M, t), a^{e}(t), e, a, t, n\right)$ where $a^{e}(t)$ is constructed in (5) and (6) and has the membership status in (7) proven by Alice. Then from the relation

$$
T E(M, t)^{e} \equiv a^{e}(t) M^{e}(\bmod n),
$$

Bob is assured that the plaintext corresponding to the RSA ciphertext $M^{e}(\bmod n)$ can be obtained from $T E(M, t)$ by performing $t$ squarings modulo $n$ starting from $a$. We should note that in this encryption scheme, Alice is the sender and Bob, the recipient; so if Alice wants the message to be timedrelease to Bob exclusively then she should send $a$ to Bob exclusively, e.g., via a confidential channel.

Remark 1. As in the case of any practical public-key encryption scheme, $M$ in (8) should be randomised using a proper plaintext randomisation scheme designed for providing the semantic security (e.g., the OAEP scheme for RSA [7]).

\subsection{Timed-Release of an RSA Signature}

Let $e, n$ be as above and $d$ satisfy $e d \equiv 1(\bmod \phi(n))$ (so $d$ is in the position of an RSA signing exponent). For message $M<n$ (see Remark 2 below), to make its RSA signature $M^{d}(\bmod n)$ releasable in time $t$, Alice can construct a "timed signature":

$$
T S(M, t) \stackrel{\text { def }}{=} a(t) M^{d}(\bmod n) .
$$

Let Bob be given the tuple $\left(M, T S(M, t), a^{e}(t), e, a, t, n\right)$ where $a^{e}(t)$ is constructed in (5) and (6) and has the membership status in (7) proven by Alice. Then from the relation

$$
T S(M, t)^{e} \equiv a^{e}(t) M(\bmod n),
$$

Bob is assured that the RSA signature on $M$ can be obtained from $T S(M, t)$ by performing $t$ squarings modulo $n$ starting from $a$. 
Remark 2. As in the case of a practical digital signature scheme, in order to prevent existential forgery of a signature, $M$ in (10) should denote an output from a cryptographically secure one-way hash function. If we further require the signature to an indistinguishability property (see §3.3), then the hashed result should be in $J_{+}(n)$. Padding $M$ with a random string and then hashing, the probability for the hashed result in $J_{+}(n)$ is 0.5 .

\subsection{Security Analysis}

Confidentiality of $\boldsymbol{M}$ in $\boldsymbol{T E}(\boldsymbol{M}, \boldsymbol{t})$ We assume that Alice has implemented properly our security requirements on the large magnitudes of $\operatorname{ord}_{\phi(n)}(2)$ and $\operatorname{ord}_{n}(a)$. Then we observe that $L(a, n)$ is a large subset of the quadratic residues modulo $n$, and the mapping $a \mapsto a(t)$ is one-way under the appropriate intractability assumption (here, integer factorisation). Consequently, our scheme for encrypting $M \in \mathbb{Z}_{n}^{*}$ in $T E(M, t)$ is a trapdoor one-way permutation since it is the multiplication, modulo $n$, of the message $M$ to the trapdoor secret $a(t)$. In fact, from (9) we see that the availability of $T E(M, t)^{e}$ and $a^{e}(t)$ makes $M^{e}$ available, and so without considering to go through $t$ squarings, the underlying intractability of $T E(M, t)$ is reduced to that of RSA. Therefore, well-known plaintext randomisation schemes for RSA encryption (e.g., OAEP [7]), which have been proposed for achieving the semantic security (against adaptive chosen ciphertext attacks) can be applied to our plaintext message before the application of the permutation. The message confidentiality properties (i.e., the indistinguishability and non-malleability on the message $M$ ) of our timed-release encryption scheme should follow directly those of RSA-OAEP.

Thus, given the difficulty of extracting the $e$-th root of a random element modulo $n$, a successful extraction of $a(t)$ from $a^{e}(t)$, or of some information regarding $M$ from $T E(M, t)$, will constitute a grand breakthrough in the area if they are done at a cost less than $t$ squarings modulo $n$.

Unforgeability of $\boldsymbol{M}^{\boldsymbol{d}}$ in $\boldsymbol{T S}(\boldsymbol{M}, \boldsymbol{t})$ First, recall that $M$ here denotes an output from a cryptographically secure one-way hash function before signing in the RSA fashion. The unforgeability of $M^{d}$ in $T S(M, t)$ follows directly that of $M^{d}(\bmod n)$ given in clear.

Secondly, the randomness of $a^{e}(t)$ ensures that of $T S(M, t)^{e}$. Thus the availability of the pair $\left(T S(M, t), T S(M, t)^{e}\right)$ does not constitute a valid signature of Alice on anything (such as on an adaptively chosen message). The availability of the pair $\left(T S(M, t), T S(M, t)^{e}\right)$ is equivalent to that of $\left(x, x^{e}\right)$ which can be constructed by anybody using a random $x$.

Indistinguishability of $\boldsymbol{M}^{d}$ in $\boldsymbol{T S}(\boldsymbol{M}, \boldsymbol{t})$ The indistinguishability is the following property: with the timed-release signature $T S(M, t)$ on $M$ and with the proven membership $a^{e}(t) \in L\left(a^{e}, n\right)$ but without going through $t$ squarings mod $n$, one should not be able to tell whether $T S(M, t)$ has any verifiable relationship with a signature on $M$. This property should hold even if the signature 
pair $\left(M, M^{d}\right)$ becomes available; namely, even if Bob has recovered the signature pair $\left(M, M^{d}\right)$ (e.g., after having performed $t$ squarings), he is still not able to convince a third party that $T S(M, t)$ is a timed-release signature of Alice on $M$. This property is shown below.

Let $\tilde{M} \in J_{+}(n)$ be any message of Bob's choice (e.g., Bob may have chosen it because $\tilde{M}^{d}$ may be available to him from a different context). We have

$$
T S(M, t) \equiv a(t) M^{d} \equiv a(t)\left(\frac{M}{\tilde{M}}\right)^{d} \tilde{M}^{d} \equiv \tilde{a} \tilde{M}^{d}(\bmod n) .
$$

So upon seeing Bob's allegation on a "verifiable relationship" between $T S(M, t)$ and $M^{d}$, the third party faces a problem of deciding which of $M^{d}$ or $\tilde{M}^{d}$ is sealed in $T S(M, t)$. This boils down to deciding if $a(t) \in L(a, n)$ or if $\tilde{a} \in L(a, n)$ (both are in $\left.J_{+}(n)\right)$, which is still a problem of going through $t$ squarings. Thus, even though the availability of $M^{d}$ and $\tilde{M}^{d}$ does allow one to recognise that the both are in fact Alice's valid signatures, without verifying the membership status, one is unable to tell if any of the two has any connection with $T S(M, t)$ at all.

\section{Membership Proof with Modulus of a Safe-Prime Structure}

Let Alice have constructed her RSA modulus $n$ with a safe-prime structure. This requires $n=p q, p^{\prime}=(p-1) / 2, q^{\prime}=(q-1) / 2$ where $p, q, p^{\prime}$ and $q^{\prime}$ are all distinct primes of roughly equal size. We assume that Alice has proven to Bob in zero-knowledge such a structure of $n$. This can be achieved via using, e.g., the protocol of Camenisch and Michels [4] 1]

Let $a \in \mathbb{Z}_{n}^{*}$ satisfy

$$
\begin{gathered}
\operatorname{gcd}(a \pm 1, n)=1, \\
\left(\frac{a}{n}\right)=-1 .
\end{gathered}
$$

It is elementary to show that a satisfying (12) and (13) has the full order $2 p^{\prime} q^{\prime}$. The following lemma observes a property of $a$.

Lemma 1. Let $n$ be an RSA modulus of a safe-prime structure and $a \in \mathbb{Z}_{n}^{*}$ of the full order. Then for any $x \in \mathbb{Z}_{n}^{*}$, either $x \in\langle a\rangle$ or $-x \in\langle a\rangle$.

Proof It's easy to check $-1 \notin\langle a\rangle$. So $\langle a\rangle$ and the coset $(-1)\langle a\rangle$ both have the half the size of $\mathbb{Z}_{n}^{*}$, yielding $\mathbb{Z}_{n}^{*}=\langle a\rangle \cup(-1)\langle a\rangle$. Any $x \in \mathbb{Z}_{n}^{*}$ is either in $\langle a\rangle$ or in $(-1)\langle a\rangle$. The latter case means $-x \in\langle a\rangle$.

\footnotetext{
${ }^{1}$ Due to the current difficulty of zero-knowledge proof for a safe-prime-structured RSA modulus, we recommend to use the method in Section 5 which works with a general form of composite modulus. The role of Section 4 is to serve a clear exposition on how we solve the current problem in timed-release cryptography.
} 


\subsection{A Building Block Protocol}

Let Alice and Bob have agreed on $n$ (this is based on Bob's satisfaction on Alice's proof that $n$ has a safe-prime structure).

Figure 1 specifies a perfect zero-knowledge protocol $(S Q)$ for Alice to prove that for $a, x, y \in \mathbb{Z}_{n}^{*}$ with $n$ of a safe-prime structure, $a$ of the full order, and $x, y \in J_{+}(n)$, they satisfy (note, \pm below means either + or - , but not both)

$$
\exists z: x \equiv \pm a^{z}(\bmod n), \quad y \equiv \pm a^{z^{2}}(\bmod n) .
$$

Alice should of course have constructed $a, x, y$ to satisfy (14). She sends $a, x, y$ to Bob.

Bob (has checked $n$ of a safe-prime structure) should first check (12) and (13) on $a$ for its full-order property (the check guarantees $a \not \equiv \pm 1(\bmod n))$; he should also check $x, y \in J_{+}(n)$.

$S Q(a, x, y, n)$

Input Common: $n$ : an RSA modulus with a safe-prime structure;

$$
\begin{array}{ll} 
& a \in \mathbb{Z}_{n}^{*}: \text { an element of the full-order } 2 p^{\prime} q^{\prime}=\phi(n) / 2 \\
& (\text { so } a \not \equiv \pm 1(\bmod n)) ; \\
& x, y \in J_{+}(n): x \not \equiv \pm y(\bmod n) ; \\
\text { Alice: } \quad & z: x \equiv \pm a^{z}(\bmod n), y \equiv \pm a^{z^{2}}(\bmod n) ;
\end{array}
$$

1. Bob chooses at random $r<n, s<n$ and sends to Alice: $C \stackrel{\text { def }}{=} a^{r} x^{s}(\bmod n)$;

2. Alice sends to Bob: $R \stackrel{\text { def }}{=} C^{z}(\bmod n)$;

3. Bob accepts if $R \equiv \pm x^{r} y^{s}(\bmod n)$, or rejects otherwise.

Fig. 1. Building Block Protocol

Remark 3. For ease of exposition this protocol appears in a non zero-knowledge format. However, the zero-knowledge property can be added to it using the notion of a commitment function: Instead of Alice sending $R$ in Step 2, she sends a commitment commit $(R)$, after which Bob reveals $r$ and $s$; this allows Alice to check the correct formation of $C$; the correct formation means that Bob has already known Alice's response.

Theorem 1. Let $a, x, y, n$ be as specified in the common input in Protocol $S Q$. The protocol has the following properties:

Completeness If the common input satisfies (14) then Bob will always accept Alice's proof; 
Soundness If (14) does not hold for the common input, then Alice, even computationally unbounded, cannot convince Bob to accept her proof with probability greater than $\frac{2 p^{\prime}+2 q^{\prime}-1}{2 p^{\prime} q^{\prime}} .2$

Zero-knowledge Bob gains no information about Alice's private input.

\section{Proof}

Completeness Evident from inspection of the protocol.

Soundness Suppose that (14) does not hold for the common input $(a, x, y, n)$ (here $x, y \in J_{+}(n)$ ) whereas Bob has accepted Alice's proof. By Lemma 1, the first congruence of (14) always holds for some $z=\log _{a} \pm x$. So it is the second congruence of (14) that does not hold for the same $z$. Let $\xi \in \mathbb{Z}_{n}^{*}$ satisfy

$$
y \equiv \xi a^{z^{2}}(\bmod n) \text { with } \xi \neq \pm 1 .
$$

Since Bob accepts the proof, he sees the following two congruences

$$
\begin{gathered}
C \equiv a^{r} x^{s}(\bmod n), \\
R \equiv \pm x^{r} y^{s}(\bmod n) .
\end{gathered}
$$

Since (16) implies

$$
C^{2} \equiv a^{2 r} x^{2 s}(\bmod n)
$$

and by Lemma 1 , both $\log _{a} C^{2}$ and $\log _{a} x^{2}\left(=\log _{a}( \pm x)^{2}=2 z\right)$ exist, we can write the following linear congruence with $r$ and $s$ as unknowns

$$
\log _{a} C^{2} \equiv 2 r+2 z s\left(\bmod 2 p^{\prime} q^{\prime}\right)
$$

For $s=1,2, \cdots, 2 p^{\prime} q^{\prime}$, this linear congruence yields $r=\frac{\log _{a} C^{2}-2 z s}{2}\left(\bmod 2 p^{\prime} q^{\prime}\right)$. Therefore there exists exactly $2 p^{\prime} q^{\prime}$ pairs of $(r, s)$ to satisfy $(16)$ for any fixed $C$ (and the fixed $a, x)$. Each of these pairs and the fixed $x, y$ will yield an $R$ from (17). Below we argue that for any two such pairs, denoted by $(r, s)$ and $\left(r^{\prime}, s^{\prime}\right)$, if $\operatorname{gcd}\left(s-s^{\prime}, 2 p^{\prime} q^{\prime}\right) \leq 2$ then they must yield $R \not \equiv \pm R^{\prime}(\bmod n)$. Suppose on the contrary for

$$
a^{r} x^{s} \equiv C \equiv a^{r^{\prime}} x^{s^{\prime}}(\bmod n), \quad \text { i.e., } \quad a^{r-r^{\prime}} \equiv x^{s^{\prime}-s}(\bmod n),
$$

it also holds

$$
x^{r} y^{s} \equiv R \equiv \pm R^{\prime} \equiv \pm x^{r^{\prime}} y^{s^{\prime}}(\bmod n), \quad \text { i.e., } \quad x^{r-r^{\prime}} \equiv \pm y^{s^{\prime}-s}(\bmod n) .
$$

Using the second congruence in (18), noticing $x \equiv \pm a^{z}$ and (15), we can transform the second congruence in (19) to

$$
( \pm 1)^{\left[r-r^{\prime}+z\left(s^{\prime}-s\right)\right]} a^{\left[z^{2}\left(s^{\prime}-s\right)\right]} \equiv x^{r-r^{\prime}} \equiv \pm y^{s^{\prime}-s} \equiv \pm \xi^{\left(s^{\prime}-s\right)} a^{\left[z^{2}\left(s^{\prime}-s\right)\right]}(\bmod n),
$$

\footnotetext{
2 The safe-prime structure of $n$ implies $p^{\prime} \approx q^{\prime} \approx \sqrt{n}$ and hence this probability value is approximately $2 / \sqrt{n}$.
} 
which yields

$$
\pm \xi^{\left(s^{\prime}-s\right)} \equiv( \pm 1)^{\left[r-r^{\prime}+z\left(s^{\prime}-s\right)\right]} \equiv \pm 1, \quad \text { i.e., } \quad \xi^{2\left(s^{\prime}-s\right)} \equiv 1(\bmod n) .
$$

Recall that $\xi \neq \pm 1$ and $y \equiv \xi a^{z^{2}} \equiv \pm \xi x^{z}(\bmod n)$ with $x, y \in J_{+}(n)$, we know $\operatorname{ord}_{n}(\xi) \neq 2$ (i.e., $\xi$ cannot be any square root of 1 , since the two roots $\neq \pm 1$ will render $\left.y \notin J_{+}(n)\right)$. Thus, $\operatorname{ord}_{n}(\xi)$ must be a multiple of $p^{\prime}$ or $q^{\prime}$ or both. However, we have assumed $\operatorname{gcd}\left(s^{\prime}-s, 2 p^{\prime} q^{\prime}\right) \leq 2$, i.e., $\operatorname{gcd}\left(2\left(s^{\prime}-s\right), 2 p^{\prime} q^{\prime}\right)=2$, so $2\left(s^{\prime}-s\right)$ cannot be such a multiple. Consequently (20) cannot hold and we reach a contradiction.

For any $s \leq 2 p^{\prime} q^{\prime}$, it's routine to check that there are $2 p^{\prime}+2 q^{\prime}-2$ cases of $s^{\prime}$ satisfying $\operatorname{gcd}\left(2\left(s^{\prime}-s\right), 2 p^{\prime} q^{\prime}\right)>2$. Thus, if (14) does not hold, amongst $2 p^{\prime} q^{\prime}$ possible $R^{\prime}$ s matching the challenge $C$, there are in total $2 p^{\prime}+2 q^{\prime}-1$ of them (matching the $s$ itself and the $2 p^{\prime}+2 q^{\prime}-2$ other $s^{\prime}$ s) that may collide to Bob's fixing of $R$. Even computationally unbounded, Alice will have at best $\frac{2 p^{\prime}+2 q^{\prime}-1}{2 p^{\prime} q^{\prime}}$ probability to have responded with a correct $R$.

Zero-Knowledge Immediate (see Remark 3).

\subsection{Proof of Membership in $L(a, n)$}

For $t \geq 1$, we can express $2^{t}$ as

$$
2^{t}= \begin{cases}2^{[2 \cdot(t / 2)]}=\left[2^{(t / 2)}\right]^{2} & \text { if } t \text { is even } \\ 2^{[2 \cdot(t-1) / 2+1]}=\left[2^{(t-1) / 2}\right]^{2} \cdot 2 & \text { if } t \text { is odd }\end{cases}
$$

Copying this expression to the exponent position of $a^{2^{t}}(\bmod n)$, we can express

$$
a^{2^{t}}(\bmod n) \equiv \begin{cases}a^{\left[2^{(t / 2)}\right]^{2}} & \text { if } t \text { is even } \\ \left(a^{\left[2^{(t-1) / 2}\right]^{2}}\right)^{2} & \text { if } t \text { is odd }\end{cases}
$$

In (21) we see that the exponent $2^{t}$ can be expressed as the square of another power of 2 with $t$ being halved in the latter. This observation suggests that repeatedly using $S Q$, we can demonstrate, in $\left\lfloor\log _{2} t\right\rfloor$ steps, that the discrete logarithm of an element is of the form $2^{t}$. This observation translates precisely into the protocol specified in Figure 2 which will terminate within $\left\lfloor\log _{2} t\right\rfloor$ steps and prove the correct structure of $a(t)$. The protocol is presented in three columns: the actions in the left column are performed by Alice, those in the right column, by Bob, and those in the middle, by the both parties.

A run of Membership $(a, t, a(t), n)$ will terminate within $\left\lfloor\log _{2} t\right\rfloor \operatorname{loops}$, and this is the completeness property. The zero-knowledge property follows that of $S Q$ (also note Remark 4(ii) below). We only have to show the soundness property.

Theorem 2. Let $n=\left(2 p^{\prime}+1\right)\left(2 q^{\prime}+1\right)$ be an RSA modulus of a safe-prime structure, $a \in \mathbb{Z}_{n}^{*}$ be of the full order $2 p^{\prime} q^{\prime}$, and $t>1$. Upon acceptance termination of Membership $(a, t, a(t), n)$, relation $a(t) \equiv \pm a^{2^{t}}(\bmod n)$ holds with probability greater than

$$
1-\frac{\left\lfloor\log _{2} t\right\rfloor\left(2 p^{\prime}+2 q^{\prime}-1\right)}{2 p^{\prime} q^{\prime}} .
$$




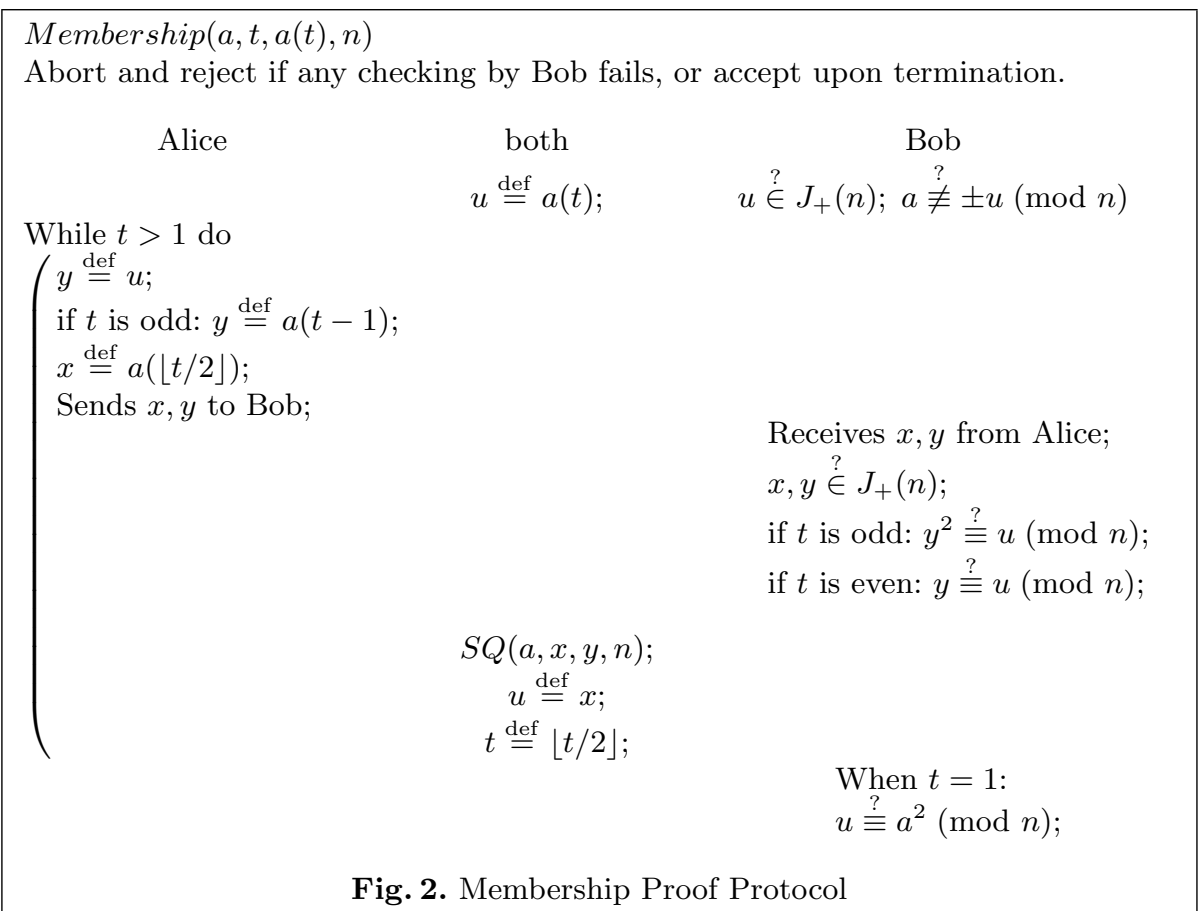

Proof Denote by $S Q\left(a, x_{1}, y_{1}, n\right)$ and by $S Q\left(a, x_{2}, y_{2}, n\right)$ any two consecutive acceptance calls of $S Q$ in Membership (so in the first call, $y_{1}=a(t)$ if $t$ is even, or $y_{1}=a(t-1)$ if $t$ is odd; and in the last call, $\left.x_{2}=a^{2}\right)$. When $t>1$, such two calls prove that there exists $z$ :

$$
x_{2} \equiv \pm a^{z}(\bmod n), y_{2} \equiv \pm a^{z^{2}}(\bmod n),
$$

and either

$$
x_{1}=y_{2} \equiv \pm a^{z^{2}}(\bmod n), y_{1} \equiv \pm a^{z^{4}}(\bmod n),
$$

or

$$
x_{1}=y_{2}^{2} \equiv a^{2 z^{2}}(\bmod n), y_{1} \equiv \pm a^{4 z^{4}}(\bmod n) .
$$

Upon $t=1$, Bob further sees that $x_{2}=a^{2}$. By induction, the exponents $z$ (resp. $\left.z^{2}, z^{4}, 2 z^{2}, 4 z^{4}\right)$ in all cases of $\pm a^{z}$ (resp. $\left.\pm a^{z^{2}}, \cdots\right)$ in (22), (23) or (24) contain a single factor: 2 . So we can write $a(t)= \pm a^{2^{u}}(\bmod n)$ for some natural number $u$.

Further note that each call of $S Q$ causes an effect of having $2^{u}$ square-rooted in the integers which is equivalent to having $u$ halved in the integers. Thus, exactly $\left\lfloor\log _{2} u\right\rfloor$ calls (and no more) of $S Q$ can be made. But Bob has counted $\left\lfloor\log _{2} t\right\rfloor$ calls of $S Q$, therefore $u=t$.

Each acceptance call of $S Q$ has the correctness probability of $1-\frac{2 p^{\prime}+2 q^{\prime}-1}{2 p^{\prime} q^{\prime}}$. So after $\left\lfloor\log _{2} t\right\rfloor$ acceptance calls of $S Q$, the probability for Membership to be 
correct is

$$
\left(1-\frac{2 p^{\prime}+2 q^{\prime}-1}{2 p^{\prime} q^{\prime}}\right)^{\left\lfloor\log _{2} t\right\rfloor}>1-\frac{\left\lfloor\log _{2} t\right\rfloor\left(2 p^{\prime}+2 q^{\prime}-1\right)}{2 p^{\prime} q^{\prime}} .
$$

Remark 4.

i) An acceptance run of Membership $(a, t, a(t), n)$ proves $\pm a(t) \in L(a, n)$, or $a^{2}(t)=a(t+1) \in L(a, n)$.

ii) It is obvious that by preparing all the intermediate values in advance, Protocol Membership can be run in parallel to save the $\left\lfloor\log _{2} t\right\rfloor$ rounds of interactions. This way of parallelisation should not be confused with another common method for parallelising a proof of knowledge protocol using a hash function to create challenge bits (which turns the proof publicly verifiable). Our parallelisation does not damage the zero-knowledge property.

iii) In most applications, $a(t)$ is the very number (solution to a puzzle) that should not be disclosed to Bob during the proof time. In such a situation, Alice should choose $t$ to be even and render $a(t-1)$ to be the solution to a puzzle. Then a proof of Membership $(a, t, a(t), n)$ will not disclose $a(t-1)$. Note that such a proof does disclose to Bob $a(\lfloor t / 2\rfloor)$ which provides Bob with a complexity of $\lfloor t / 2\rfloor-1$ squarings to reach $a(t-1)$. To compensate the loss of computation, proof of Membership $(a, 2 t, a(2 t), n)$ is necessary. Consequently, the proof runs one loop more than $\operatorname{Membership}(a, t, a(t), n)$ does. Note that the above precautions are unnecessary for our applications in $\S 3$ where it is the $e$-th root of $a^{e}(t)$ that is the puzzle's solution; the disclosures of $a^{e}(t)$ or $a^{e}(\lfloor t / 2\rfloor)$ do not seem to reduce the time complexity for finding $a(t)$.

\subsection{Performance}

In each run of $S Q$, Alice (resp. Bob) performs one (resp. four) exponentiation(s) $\bmod n$. So in Membership $(a, t, a(t), n)$ Alice (resp. Bob) will perform $\left\lfloor\log _{2} t\right\rfloor$ (resp. $\left.4\left\lfloor\log _{2} t\right\rfloor\right)$ exponentiations $\bmod n$. These translate to $O\left(\left\lfloor\log _{2} t\right\rfloor\left(\log _{2} n\right)^{3}\right)$ bit operations.

In the LCS35 Time Capsule Crypto-Puzzle [10], $t=79685186856218$ is a 47-bit binary number. Thus the verification for that puzzle can be completed within $4 \times 47=188$ exponentiations $\bmod n$.

The number of bits to be exchanged is measured by $O\left(\left(\left\lfloor\log _{2} t\right\rfloor\right)\left(\log _{2} n\right)\right)$.

\section{Use of Modulus of a General Form}

When $n$ does not have a safe-prime structure, the error probability of $S Q$ can be much larger than what we have measured in Theorem 1 . The general method for Alice to introduce an error in her proof (i.e., to cheat) is to fix $y$ in (15) with some $\xi \neq \pm 1$. For $y$ so fixed before Bob's challenge $C$, Bob is actually awaiting for $R \equiv \pm C^{z} \xi^{s}(\bmod n)$ in which $\xi^{s}(\bmod n)$ is the only value that 
Alice does not know (she does not know it because Bob's random choice of $s$ is perfectly hidden in $C$ ). Therefore in order to respond with the correct $R$, it is both necessary and sufficient for Alice to guess $\xi^{s}(\bmod n)$ correctly. Notice that while it is unnecessary and can be too difficult for Alice to guess $s$, guessing $\xi^{s}(\bmod n)$ need not be very difficult and the probability of a correct guess is bounded by $\frac{1}{\operatorname{ord}_{n}(\xi)}$. Thus, in order for Alice to achieve a large error probability (meaning, to ease her cheating), she should use $\xi$ of a small order.

The above cheating scenario provides the easiest method for Alice to cheat and yet is general enough for covering the cases that the soundness of $S Q$ should consider. Multiplying both $x$ and $y$ with some small-order elements will only make the cheating job more difficult. Therefore it suffices for us to anticipate the above general cheating method.

To this end it becomes apparent that in order to limit Alice's cheating probability we should prevent her from constructing $y$ in (15) using $\xi$ of a small order. Using a safe-prime-structured modulus $n=\left(2 p^{\prime}+1\right)\left(2 q^{\prime}+1\right)$ achieves this purpose exactly because then the least order available to Alice is $\min \left(\frac{1}{p^{\prime}}, \frac{1}{q^{\prime}}\right)$ which is satisfiably small (using $\xi$ of order 2 either does not constitute an attack, or will cause detection of $\left.y \notin J_{+}(n)\right)$.

While a zero-knowledge proof of $n$ being in a safe-prime structure is computationally inefficient to date, it is rather easy to construct a zero-knowledge proof protocol for proving that $\phi(n)$ is free of small odd prime factors up to a bound $B$. Boyar et al [2] constructed a practically efficient zero-knowledge proof protocol for proving that $\phi(n)$ is relatively prime to $n$. As in [8], we can apply the same idea to prove that $\phi(n)$ is relatively prime to $\Delta$ (i.e., using $\Delta$ in place of $n$ ) where

$$
\Delta=\prod_{\substack{\text { primes } \ell: \\ 2<\ell<B}} \ell .
$$

Supposing that $n$ is a Blum integer (which can be efficiently proved using, e.g., the protocol of van de Graaf and Peralta [12]), then after applying the protocol of Boyar et al using $\Delta$ in (25) in place of $n$, we can be sure that the error probability of $S Q$ is bounded by $B^{-1}$. Notice that the multiplication attack using the square roots of 1 with the negative Jacobi symbol (in place of $\xi$ in (15)) is not possible since that will be detected by the Jacobi symbol checking conducted on the input values. Thus, if Alice is required to repeat running $S Q$ $\frac{k}{\log _{2} B}$ times, then Bob is sure that her cheating probability (i.e., for (14) not to hold) is bounded by $2^{-k}$.

\subsection{Performance of Membership Proof Using General Form of Modulus}

With the soundness probability of $S Q$ bounded by $B^{-1}$, for each case of $x, y$, $S Q(a, x, y, n)$ need to be run $\frac{k}{\log _{2} B}$ times to achieve an acceptable soundness probability $2^{-k}$. Thus in Membership, $S Q$ is run $\frac{\left\lfloor\log _{2} t\right\rfloor k}{\log _{2} B}$ times. Since in each 
run of $S Q$, Alice (resp. Bob) performs one (resp. four) exponentiation(s) mod $n$. So in Membership $(a, t, a(t), n)$ Alice (resp. Bob) will perform $\frac{\left\lfloor\log _{2} t\right\rfloor k}{\log _{2} B}$ (resp. $\frac{4\left\lfloor\log _{2} t\right\rfloor k}{\log _{2} B}$ exponentiations mod $n$. Adding to this is the cost for running $k$ times the protocol of Boyar et al, each run of that protocol costs one modulo exponentiation for both parties. Thus, the total cost in number of exponentiations mod $n$ of the membership proof for Alice is

$$
\frac{\left\lfloor\log _{2} t\right\rfloor k}{\log _{2} B}+k,
$$

and that for Bob is

$$
\frac{4\left\lfloor\log _{2} t\right\rfloor k}{\log _{2} B}+k .
$$

In the LCS35 Time Capsule Crypto-Puzzle [10] where $\left\lfloor\log _{2} t\right\rfloor=47$, if we consider $B=2^{10}$ and $k=100$, then the quantity for Alice is 570 and that for Bob is 1980. Therefore, the LCS35 Time Capsule Crypto-Puzzle using a general-form modulus (Blum integer) can be verified with 1980 modulo exponentiations.

Zero-knowledge proof of a Blum integer using the protocol in [12] has a performance similar to one modulo exponentiation for Alice; the workload of that protocol for Bob is trivial since it only involves multiplications and evaluations of Jacobi symbols. Thus, considering the same low soundness probability of $2^{-100}$, we should add 100 modulo exponentiations to Alice's workload to reach 670 modulo exponentiations.

\section{Conclusion}

We have constructed an efficient zero-knowledge protocol for providing general solutions to timed-release cryptographic problems (encryption and signature). These schemes have proven correctness on time control which can be fine tuned to the granularity in number of multiplications.

Successful timed-release cryptographic problems have been constructed upon the integer-factoring based intractability. An important feature that such intractability offers is non-parallelisability. An open question is that can other intractability offer this feature? (We know that the problem of extraction of discrete logarithm can be parallelised [13.)

\section{Acknowledgments}

I would like to thank Steven Galbraith, Kenny Paterson, David Soldera and the anonymous referees of SAC'01 for their helpful comments on a draft of this paper. 


\section{References}

1. Blum, M. Coin Flipping by Telephone: A Protocol for Solving Impossible Problems, Proceedings of the 24th IEEE Computer Conference, pages 133-137, 1981.

2. Boyar, J., Friedl, K. and Lund, C. Practical zero-knowledge proofs: Giving hints and using deficiencies, Advances in Cryptology - Proceedings of EUROCRYPT 89 (J.-J. Quisquater and J. Vandewalle, eds.), Lecture Notes in Computer Science 434, Springer-Verlag 1990, pages 155-172.

3. Boneh, D. and Naor, M. Timed commitments (extended abstract), Advances in Cryptology: Proceedings of CRYPTO'00, Lecture Notes in Computer Science 1880, Springer-Verlag 2000, pages 236-254.

4. Camenisch J. and Michels, M. Proving in zero-knowledge that a number is the product of two safe primes, In Advances in Cryptology - EUROCRYPT 99 (J. Stern ed.), Lecture Notes in Computer Science 1592, Springer-Verlag 1999, pages $106-121$.

5. Chaum, D. Zero-knowledge undeniable signatures, Advances in Cryptology: Proceedings of CRYPTO 90 (I.B. Damgaard, ed.) Lecture Notes in Computer Science 473, Springer-Verlag 1991, pages 458-464.

6. Damgård, I. Practical and probably secure release of a secret and exchange of signatures, Advances in Cryptology - Proceedings of EUROCRYPT 93 (T. Helleseth ed.), Lecture Notes in Computer Science 765, Springer-Verlag 1994, pages 200-217.

7. Fujisaki, E., Okamoto, T. Pointcheval, D. and Stern, J. RSA-OAEP is Secure under the RSA Assumption, To appear in Advances in Cryptology: Proceedings of CRYPTO 01, Springer-Verlag 2001.

8. Galbraith, S., Mao, W. and Paterson, K. RSA-based undeniable signatures for general moduli, to appear in the 2002 RSA Conference, Cryptographers' Track, February 2002.

9. Goldreich, O., Micali, S. and Wigderson, A. How to prove all NP statements in zero-knowledge and a methodology of cryptographic protocol design, Advances in Cryptology - Proceedings of CRYPTO 86 (A.M. Odlyzko ed.), Lecture Notes in Computer Science, Springer-Verlag 263 (1987), pages 171-185.

10. Rivest, R.L. Description of the LCS35 Time Capsule Crypto-Puzzle, http://www.lcs.mit.edu/about/tcapintro041299, April 4th, 1999.

11. Rivest, R.L., Shamir, A. and Wagner, D.A. Time-lock puzzles and timed-release crypto, Manuscript. Available at (http://theory.lcs.mit.edu/ ${ }^{\sim}$ rivest/RivestShamirWagner-timelock.ps).

12. van de Graaf, J. and Peralta, R. A simple and secure way to show that validity of your public key, (C. Pomerance ed.), CRYPTO '87, Springer LNCS 293, (1988) $128-134$.

13. van Oorschot, P.C. and Wiener, M.J. Parallel collision search with cryptanalytic applications, J. of Cryptology, Vol.12, No.1 (1999), pages 1-28. 